\title{
EIGENVALUE METHOD AS A FULL MEASURING TOOL
}

\author{
I. L. Tomashevskii \\ Institute of Mathematics, Information and Space Technologies \\ Northern (Arctic) Federal University \\ Arkhangelsk, Russia \\ E-mail: tomashevil@gmail.com
}

\begin{abstract}
The eigenvalue method (EM) is a well-known approach to deriving information from pairwise comparison matrices in Analytic Hierarchy Process. However this method isn't logically complete since its actual numerical error is unknown and its robustness is doubted by the problems of EM, such as "right-left asymmetry", "rank reversal", and violation of "order of preference" and "order of intensity of preference".

We show that EM, as a calculation procedure, is equivalent to some «matrix» measuring process. This process is analyzed from the viewpoint of measurement theory. Formulas for the actual EM error are obtained and it is shown that the problems of EM are eliminated when we take into account the numerical values of the EM error.

The full measuring tool is composed of pairwise comparisons as a measuring procedure, EM as a data processor, and the obtained formulas as an error indicator. This tool is suitable to measure and ranking any comparable elements with positive numerical values. The mean relative error of the tool is equal to the square root of the double Saaty's Consistency Index. In the case of decision making processes, the numerical value of errors entirely depends on a measuring scale inaccuracy and inconsistency of expert judgements.
\end{abstract}

Keywords: pairwise comparisons, eigenvalue method, Analytic Hierarchy Process, decision analysis.

\section{Introduction}

The eigenvalue method (EM) is a handy tool to deriving information from pairwise comparison matrices. This method proposed by Saaty (1977) for the Analytic Hierarchy Process (AHP) derives values (priorities) $\boldsymbol{\omega}_{1}, \boldsymbol{\omega}_{2}, . ., \boldsymbol{\omega}_{h}$ of comparable elements $\Omega_{1}, \Omega_{2}, \ldots, \Omega_{n}$ as the solution of the right eigenvector problem

$$
A\left(\begin{array}{c}
\omega_{1} \\
\ldots \\
\omega_{n}
\end{array}\right)=\lambda_{\max }\left(\begin{array}{c}
\omega_{1} \\
\ldots \\
\omega_{n}
\end{array}\right)
$$

for the corresponding pairwise comparison matrix $A$ with principal eigenvalue $\lambda_{\max }$. This elegance and versatile method is widely applied for analysis of different practical problems. However, EM isn't logically complete. Firstly, the actual numerical error of the derived values $\boldsymbol{\omega}_{1}, \boldsymbol{\omega}_{2}, \ldots, \boldsymbol{\omega}_{h}$ is unknown. The values $\omega_{1}, \omega_{2}, . ., \omega_{n}$ are exact only when the pairwise comparison matrix $A$ is perfectly consistent. In the inconsistent case, the Consistency Ratio (CR) (Saaty, 1980) and 
Saaty's criterion of $C R \leq 0.1$ is used to accept or reject a comparison matrix. The eligibility of this criterion has been much debated. Regardless of the opinions, $C R$ is a heuristic criterion, which does not purpose to detect actual EM error. Secondly, Johnson et al. (1979) show a rank reversal problem for scale inversion: the ranking of elements depends on the formulation of the problem. Thirdly, one of the controversial aspects of AHP and EM is the rank reversal phenomenon caused by the addition or deletion of an element. The famous example of rank reversal in EM is demonstrated by Hochbaum et al. (2006). Fourthly, EM allows the violation "order of intensity of preference" (Bana e Costa and Vansnick, 2008).

Our aim is to find the actual EM error, to show as the above problems are selfeliminated when we take into account the numerical values of the EM error, and to show that EM can be considered as a full measuring tool.

\section{EM as a measuring tool}

We show that EM, as a calculation procedure, is equivalent to some «matrix» measuring procedure for comparable elements $\Omega_{1}, \Omega_{2}, \ldots, \Omega_{n}$ :

$$
\left\{\begin{array}{c}
\Omega_{1} \\
\ldots \\
\Omega_{n}
\end{array}\right\} \rightarrow\left\langle\begin{array}{c}
\text { measuring } \\
\text { procedure }
\end{array}\right\rangle \rightarrow\left\{\begin{array}{c}
\omega_{1}^{(1)}, . ., \omega^{(n)} \\
\ldots \\
\omega_{n}^{(1)}, . ., \omega_{n}^{(n)}
\end{array}\right\} .
$$

This procedure is analyzed from the viewpoint of measurement theory. Formulas for the actual EM errors $\Delta \omega_{1}, . ., \Delta \omega_{h}$ are obtained. We show that these formulas together with pairwise comparison procedure and the EM algorithm (1) compose the full measuring tool to measure and rank any comparable elements $\Omega_{1}, \Omega_{2}, \ldots, \Omega_{n}$ with positive numerical values:

$$
\left\{\begin{array}{c}
\Omega_{1} \\
\ldots \\
\Omega_{n}
\end{array}\right\} \rightarrow\left\langle\begin{array}{c}
\text { measuring } \\
\text { tool }
\end{array}\right) \rightarrow\left\{\begin{array}{c}
\omega_{1} \pm \Delta \omega_{1} \\
\ldots \\
\omega_{n} \pm \Delta \omega_{n}
\end{array}\right\}
$$

Also we consider the other version of this tool, which uses the algorithm (1) for transposed matrix $A^{T}$, i.e., for left principal eigenvector of $A$. In the case absolutely precise measurements, this version generates inverse values of the compared elements. For both versions, the mean relative error is equal to

$$
\left(\frac{\Delta \omega_{i}}{\omega_{i}}\right)_{\text {mean }} \equiv \sqrt{\frac{1}{n} \sum_{k=1}^{n}\left(\frac{\Delta \omega_{k}}{\omega_{k}}\right)^{2}} \approx \sqrt{2 \frac{\lambda_{\max }-n}{n-1}},
$$

and for any $3 \times 3$ reciprocally symmetric comparison matrices

$$
\frac{\Delta \omega_{1}}{\omega_{1}}=\frac{\Delta \omega_{2}}{\omega_{2}}=\frac{\Delta \omega_{3}}{\omega_{3}}=\left(\frac{\Delta \omega_{i}}{\omega_{i}}\right)_{\text {mean }} .
$$

Both tools can be used equally to ranking elements $\Omega, \Omega_{2}, \ldots, \Omega_{n}$.

\section{On criticism of EM}


These tools are used for the analysis of the above EM problems. We test different phenomena, which suggest that the EM ranking isn't reliable. These phenomena are connected with two principal facts. First, the pairwise comparisons of elements under consideration are not consistent. Second, only ranking of mean values $\boldsymbol{C Q P}_{,}, ., \boldsymbol{C} \boldsymbol{h}$ is used for ranking of elements $\Omega_{1}, \Omega_{2}, \ldots, \Omega_{n}$. The last is especially important because in the case

$$
\Delta \omega_{k}+\Delta \omega_{i}>3\left|\omega_{k}-\omega_{i}\right|
$$

the inequality $\omega_{k}>\omega_{i}$ (or $\omega_{k}<\omega_{i}$ ) does not contain any information on the ranking of the elements $\Omega_{k}$ and $\Omega_{i}$, moreover the ranking loses any sense. We show that all "problematic" examples (Johnson et al.,1979; Saaty, 1980; Hochbaum et al., 2006, and other) correspond to such EM error that (2) holds. The EM problems are selfeliminated.

\section{REFERENCES}

Bana e Costa, C.A., Vansnick, J.-C. (2008) A critical analysis of the eigenvalue method used to derive priorities in AHP. European Journal of Operational Research, 187, 1422-1428.

Hochbaum D.S. and Levin A. (2006) Methodologies for the group rankings decision. _Management Science, 52, 1394-1408.

Johnson, C., Beine, W., \& Wang, T. (1979) Right-Left Asymmetry in an Eigenvector Ranking Procedure. Journal of Mathematical Psychology, 19, 61-64.

Saaty, T.L. (1977) A scaling method for priorities in hierarchical structures. Journal of Mathematical Psychology, 15, 234-281.

Saaty, T.L. (1980) The Analytic Hierarchy Process. New York: McGraw Hill. 